\title{
Consensus Problem of High-Order Multiagent Systems with Time Delays
}

\author{
Jianhui Liu ${ }^{1}$ and Bin Zhang ${ }^{2}$ \\ ${ }^{1}$ School of Automation Science and Electrical Engineering, Beihang University, Beijing 100191, China \\ ${ }^{2}$ School of Automation, Beijing University of Posts and Telecommunications, Beijing 100876, China \\ Correspondence should be addressed to Bin Zhang; zb362301@126.com
}

Received 5 December 2016; Revised 10 February 2017; Accepted 16 February 2017; Published 2 March 2017

Academic Editor: Mohammad D. Aliyu

Copyright (c) 2017 Jianhui Liu and Bin Zhang. This is an open access article distributed under the Creative Commons Attribution License, which permits unrestricted use, distribution, and reproduction in any medium, provided the original work is properly cited.

In this paper, we consider the consensus problem of high-order multiagent systems on both fixed and switching interaction topologies with time delays. A neighbor-based protocol is presented, under which we prove that the state errors converge to zero asymptotically if there is a solution to a given Riccati inequality. The proof of our theorem is shown in time domain based on a Lyapunov approach. A numerical example is introduced to indicate the correctness of our analysis.

\section{Introduction}

Multiagent systems have aroused general interest as an area of research. Multiagent system is a network system, under distributed control protocols, consisting of large amounts of simple individuals which interact with each other. The agents, via limited communication topology, work together to accomplish tasks that each individual cannot achieve. This research topic is widely used in our daily lives, such as consensus problems, flocking of insects, and design of sensor networks. In particular, the objective of consensus problem is to design distributed control laws for the agents, using local information from their neighbors, such that the states of the agents converge to an agreement.

Many notable results have been obtained for multiagent coordination in the past decades. Vicsek et al. [1] presented a discrete time model of multiagent system and a local information protocol. Jadbabaie et al. [2] gave a strict proof of Vicsek model, in which graph theory was introduced to describe the interaction of the agents. Olfati-Saber and Murray [3] analyzed consensus problem of continuous time model and obtained great results which were extended by Ren and Beard [4]. Based on the study above, scholars addressed consensus problem with time delays $[5,6]$ and external disturbances $[7,8]$. Based on consensus schemes, necessary and sufficient graphical conditions for formation control of unicycles were discussed by Lin et al. [9]. In the analysis above, the agents are identical, but nature has presented examples of collective behavior in group with a leader such as fish and birds. Motivated by the nature phenomena, Couzin et al. [10] revealed the law of collective behavior with a leader through experiments. Hu and Hong [11] discussed a leader-following consensus problem for multiple agents with coupling time delays. Hong et al. [12] considered a leaderfollowing consensus problem with jointly connected topologies by a Lyapunov-based approach. Zheng and Wang [13] considered the consensus problem of switched multiagent system composed of continuous time and discrete time subsystems. Lin and Zheng [14] considered the finite time consensus problem of switched multiagent system which is composed of continuous time and discrete time subsystems. However, most of the existing studies focus on analysis of first-order or second-order multiagent systems. There are few results about consensus problem of high-order multiagent systems with switching topology, and time delays.

In this paper, we consider the consensus problem of highorder multiagent systems with switching topology and time delays by a leader-following approach. We use the state of the leader as a dynamic target of the system and the state of the leader is independent of others. Limited by the constraints 
on measurement, the dynamic target cannot be achieved by some agents or all the agents in some time intervals. In this situation, we only assume the system to be jointly connected, which is a much weaker assumption than that of Hong et al. [12]. This paper is organized as follows. In Section 2, preliminaries and problem formulation are presented. Fixed topology case with time delays and switching topology case with time delays are analyzed in Sections 3 and 4, respectively. Finally, Section 5 presents simulations and Section 6 gives a conclusion of this paper.

\section{Preliminaries and Problem Formulation}

2.1. Graph Theory. In this section, we first introduce some definitions and notations in graph theory. Let $\mathscr{G}$ be an undirected graph of order $n$ with the set of nodes $v=$ $\left\{v_{1}, v_{2}, \ldots, v_{n}\right\}$ and edges $\varepsilon \subseteq \nu \times \nu$. Because of the fact that $\mathscr{G}$ is undirected, $\left(v_{i}, v_{j}\right) \in \varepsilon$ if and only if $\left(v_{j}, v_{i}\right) \in \varepsilon$. The adjacency matrix $A=\left[a_{i j}\right]$ is defined as $a_{i i}=0$ and $a_{i j}=a_{j i} \geq 0$, where $a_{i j}>0$ if and only if $\left(\nu_{i}, v_{j}\right) \in \varepsilon$. The Laplacian matrix of graph $\mathscr{G}$ is defined as $L=D-A$, where $D=\operatorname{diag}\left\{d_{1}, d_{2}, \ldots, d_{n}\right\}$ is a diagonal matrix with diagonal elements $d_{i}=\sum_{j=1}^{n} a_{i j}$ for $i=1,2, \ldots, n$. The set of neighbors of node $v_{i}$ is denoted by $\mathcal{N}_{i}=\left\{v_{j} \in \nu:\left(v_{i}, v_{j}\right) \in \varepsilon\right\}$. If there is a path between any two nodes of the graph $\mathscr{G}$, then $\mathscr{G}$ is said to be connected. A component of graph $\mathscr{G}$ is defined as a connected subgraph that is maximal. The union of a collection of graphs $\mathscr{G}_{1}, \ldots, \mathscr{G}_{m}$, with the same node set $v$, is defined as the graph $\mathscr{G}_{\widehat{m}}$ with the node set given by $v$ and the edge set given by the union of the edge sets of all the graphs in the collection.

In this paper, we focus on a system consisting of a leader and $n$ agents. Graph theory is used to describe the information exchange between the leader and the agents. $\overline{\mathscr{G}}$ is defined as the graph associated with the system. Obviously, $\overline{\mathscr{G}}$ consists of the leader, edges between the leader and its neighbors, and $\mathscr{G}$ which is the graph of the $n$ agents. Graph $\overline{\mathscr{G}}$ is said to be connected if at least one agent in each component of $\mathscr{G}$ is connected to the leader.

2.2. Consensus Protocol. Consider that a multiagent system consists of $N$ agents and a leader. The dynamics of each agent, labeled from 1 to $N$, are described as

$$
\dot{x}_{i}=A x_{i}+B u_{i}, \quad i=1, \ldots, N,
$$

where $x_{i} \in R^{n}$ is the state associated with the $i$ th agent and $u_{i} \in R^{m}$ is the input of the $i$ th agent which can only take feedback information from its neighbors. The dynamics of the leader, labeled as 0 , are described as

$$
\dot{x}_{0}=A x_{0}+B u_{0}
$$

where $u_{0}$ is the given input which determines the objective trajectory. Different from the results of [8], where secondorder multiagent systems are considered, in this paper, we investigate high-order multiagent systems.
Definition 1. The leader-following consensus problem is said to be solved if and only if the agents with local state feedback satisfy

$$
\lim _{t \rightarrow \infty}\left\|x_{i}(t)-x_{0}(t)\right\|=0
$$

for any initial condition $x_{i}(0), i=0,1, \ldots, N$.

In this paper, we use the following consensus protocol:

$$
\begin{aligned}
u_{i}(t)= & u_{0}(t)+\sum_{j \in N_{i}} a_{i j} K\left[x_{j}(t-\tau)-x_{i}(t-\tau)\right] \\
& +e_{i} K\left[x_{0}(t-\tau)-x_{i}(t-\tau)\right],
\end{aligned}
$$

where $K$ is a feedback matrix, $a_{i j}>0$ and $e_{i}>0$ are the adjacency weights of graph $\overline{\mathscr{G}}$, and $\tau>0$ is the time delay. We denote the $i$ th state error as $\varepsilon_{i}=x_{i}-x_{0}$. With feedback (4), the closed-loop system can be summarized as

$$
\dot{\xi}(t)=\left(I_{N} \otimes A\right) \xi(t)-[(L+E) \otimes B K] \xi(t-\tau),
$$

where $\xi=\left(\varepsilon_{1}^{T}, \ldots, \varepsilon_{N}^{T}\right)^{T}, E=\operatorname{diag}\left\{e_{1}, \ldots, e_{N}\right\}$ and $L$ is the Laplacian matrix. In the following, we define $M=L+E$ as the information exchange matrix. Furthermore, we define the maximum eigenvalue of $M$ as $\delta$ and the minimum eigenvalue of $M$ as $\eta$.

\section{Formation under Fixed Topology}

In this section, we focus on the leader-following consensus problem of multiagent systems with fixed interaction topology. Before presenting our main results, we will introduce the following lemma first.

Lemma 2 (see [12]). If graph $\overline{\mathscr{G}}$ is connected, then the symmetric matrix $M$ associated with $\overline{\mathscr{G}}$ is positive definite.

With Lemma 2, we can see that if graph $\overline{\mathscr{G}}$ is connected, then the minimum eigenvalue of $M$ is positive; that is, $\eta>$ 0 . Now we are in position to present our main result in this section.

Theorem 3. Consider the multiagent system (1)-(2). If graph $\bar{G}$ is connected and there exists a matrix $P>0$ such that

$$
P A+A^{T} P+\left(1+\delta^{2}\right) P B B^{T} P+\eta I<0,
$$

then the leader-following consensus problem can be solved under control protocol (4), where $K=B^{T} P$ is the feedback matrix.

Proof. Let $P>0$ be a solution of the Riccati equation (6) and $K=B^{T} P$. We take the following Lyapunov function:

$$
\begin{aligned}
V(t)= & \xi^{T}(t)\left(I_{N} \otimes P\right) \xi(t) \\
& +\int_{t-\tau}^{t} \xi^{T}(s)\left(I_{N} \otimes P B B^{T} P\right) \xi(s) d s .
\end{aligned}
$$


The derivative of $V(t)$ is

$$
\begin{aligned}
\dot{V}(t)= & 2 \xi^{T}(t)\left(I_{N} \otimes P\right) \\
& \cdot\left[\left(I_{N} \otimes A\right) \xi(t)-\left(M \otimes B B^{T} P\right) \xi(t-\tau)\right] \\
+ & \xi^{T}(t)\left(I_{N} \otimes P B B^{T} P\right) \xi(t)-\xi^{T}(t-\tau) \\
& \cdot\left(I_{N} \otimes P B B^{T} P\right) \xi(t-\tau) .
\end{aligned}
$$

Based on Lemma 2, we have that matrix $M$ is positive definite. Thus there exists an orthogonal matrix $U \in R^{N \times N}$ such that

$$
U^{T} M U=\operatorname{diag}\left\{\lambda_{1}, \ldots, \lambda_{N}\right\}
$$

where $\lambda_{i}>0, i=1, \ldots, N$. Let $\bar{\xi}(t)=\left(U^{T} \otimes I_{n}\right) \xi(t)$. Then (8) can be rewritten as

$$
\begin{aligned}
& \dot{V}(t)=2 \bar{\xi}^{T}(t)\left[\left(I_{N} \otimes P A\right) \bar{\xi}(t)-\left(U^{T} M U \otimes P B B^{T} P\right)\right. \\
& \cdot \bar{\xi}(t-\tau)]+\bar{\xi}^{T}(t)\left(I_{N} \otimes P B B^{T} P\right) \bar{\xi}(t)-\bar{\xi}^{T}(t-\tau) \\
& \cdot\left(I_{N} \otimes P B B^{T} P\right) \bar{\xi}(t-\tau) \\
& =\sum_{i=1}^{N}\left[\bar{\xi}_{i}^{T}(t)\left(P A+A^{T} P+P B B^{T} P\right) \bar{\xi}_{i}(t)\right. \\
& -2 \lambda_{i} \bar{\xi}_{i}^{T}(t) P B B^{T} P \bar{\xi}_{i}(t-\tau) \\
& \left.-\bar{\xi}_{i}^{T}(t-\tau) P B B^{T} P \bar{\xi}_{i}(t-\tau)\right] .
\end{aligned}
$$

Note that, for any vector $a, b \in R^{n}$ and any positive definite matrix $X \in R^{n \times n}$,

$$
-2 a^{T} b \leq \inf _{X>0}\left\{a^{T} X a+b^{T} X^{-1} b\right\} .
$$

Then we can obtain that

$$
\begin{aligned}
-2 \lambda_{i} \bar{\xi}_{i}^{T}(t) P B B^{T} P \bar{\xi}_{i}(t-\tau) \\
\leq \lambda_{i}^{2} \bar{\xi}_{i}^{T}(t) P B B^{T} P \bar{\xi}_{i}(t) \\
\quad+\bar{\xi}_{i}^{T}(t-\tau) P B B^{T} P \bar{\xi}_{i}(t-\tau) .
\end{aligned}
$$

Therefore, we have

$$
\begin{aligned}
\dot{V}(t) & \leq \sum_{i=1}^{N} \bar{\xi}_{i}^{T}(t)\left[P A+A^{T} P+\left(1+\lambda_{i}^{2}\right) P B B^{T} P\right] \bar{\xi}_{i}(t) \\
& \leq \sum_{i=1}^{N} \bar{\xi}_{i}^{T}(t)\left[P A+A^{T} P+\left(1+\delta^{2}\right) P B B^{T} P\right] \bar{\xi}_{i}(t) \\
& \leq-\eta \sum_{i=1}^{N} \bar{\xi}_{i}^{T}(t) \bar{\xi}_{i}(t)=-\eta \sum_{i=1}^{N} \xi_{i}^{T}(t) \xi_{i}(t)
\end{aligned}
$$

which completes the proof.

\section{Formation under Switching Topology}

In this section, we extend our result to the leader-following consensus problem of multiagent system with switching topology. In the switching topology case, the neighbors of each agent change with respect to time. To describe the variable topology, we define $\sigma:[0, \infty) \rightarrow \mathscr{P}$ as a switching signal, where $\mathscr{P}$ is a finite index set. Similar to (5), the closedloop system in the switching topology case can be written as

$$
\begin{aligned}
\dot{\xi}(t) & =\left(I_{N} \otimes A\right) \xi(t)-\left[\left(L_{\sigma(t)}+E_{\sigma(t)}\right) \otimes B K\right] \xi(t-\tau) \\
& =\left(I_{N} \otimes A\right) \xi(t)-\left(M_{\sigma(t)} \otimes B K\right) \xi(t-\tau) .
\end{aligned}
$$

Consider an infinite sequence of nonempty, bounded, and contiguous time intervals $\left[t_{i}, t_{i+1}\right), i=0,1, \ldots$, with $t_{0}=0$ and $t_{i+1}-t_{i} \leq T$, for some constant $T>0$. In each interval $\left[t_{i}, t_{i+1}\right)$, there is a sequence of nonoverlapping subintervals:

$$
\left[t_{i}^{0}, t_{i}^{1}\right),\left[t_{i}^{1}, t_{i}^{2}\right), \ldots,\left[t_{i}^{m_{i}-1}, t_{i}^{m_{i}}\right)
$$

with $t_{i}^{0}=t_{i}$ and $t_{i}^{m_{i}}=t_{i+1}$. The communication topology $M_{\sigma(t)}$ switches at $t_{i}^{j}$. Suppose that $t_{i}^{j+1}-t_{i}^{j} \geq \tau, 0 \leq j \leq$ $m_{i}-1$, and the topology does not change during each time interval $\left[t_{i}^{j}, t_{i}^{j+1}\right)$. The graphs are said to be jointly connected across time interval $\left[t_{i}, t_{i+1}\right)$ if the union of graphs $\left\{\overline{\mathscr{G}}_{\sigma(t)}: t \in\right.$ $\left.\left[t_{i}, t_{i+1}\right)\right\}$ is jointly connected.

In the time interval $\left[t_{i}, t_{i+1}\right)$, we define $\delta_{j}$ as the maximum eigenvalue of $M_{\sigma\left(t_{i}^{j}\right)}$ and $\eta_{j}$ as the minimum eigenvalue of $M_{\sigma\left(t_{i}^{j}\right)}$, where $0 \leq j \leq m_{i}-1$. Let $\delta=\max \left\{\delta_{j}, 0 \leq\right.$ $\left.j \leq m_{i}-1\right\}, \bar{\delta}>\delta$, where $\bar{\delta}$ is a given constant and $\eta=$ $\max \left\{\eta_{j}, 0 \leq j \leq m_{i}-1\right\}$.

Theorem 4. Consider the multiagent system (1)-(2). If the graphs are jointly connected across each time interval $\left[t_{i}, t_{i+1}\right), i=0,1, \ldots$, and there exists a matrix $P>0$ such that

$$
P A+A^{T} P+\left(1+\bar{\delta}^{2}\right) P B B^{T} P+\eta I<0,
$$

then the leader-following consensus problem can be solved under control protocol (4), where $K=B^{T} P$ is the feedback matrix.

Proof. Let $P>0$ be a solution of the Riccati equation (16) and $K=B^{T} P$. We take the following Lyapunov function:

$$
\begin{aligned}
V(t)= & \xi^{T}(t)\left(I_{N} \otimes P\right) \xi(t) \\
& +\int_{t-\tau}^{t} \xi^{T}(s)\left(I_{N} \otimes P B B^{T} P\right) \xi(s) d s .
\end{aligned}
$$

In each time interval $\left[t_{i}^{j}, t_{i}^{j+1}\right)$, the derivative of $V(t)$ is

$$
\begin{aligned}
\dot{V}(t)= & 2 \xi^{T}(t)\left(I_{N} \otimes P\right) \\
& \cdot\left[\left(I_{N} \otimes A\right) \xi(t)-\left(M_{\sigma} \otimes B B^{T} P\right) \xi(t-\tau)\right] \\
+ & \xi^{T}(t)\left(I_{N} \otimes P B B^{T} P\right) \xi(t)-\xi^{T}(t-\tau) \\
& \cdot\left(I_{N} \otimes P B B^{T} P\right) \xi(t-\tau) .
\end{aligned}
$$


Since the matrix $M_{\sigma}$ is positive semidefinite, there exists an orthogonal matrix $U_{\sigma} \in R^{N \times N}$ such that

$$
U_{\sigma}^{T} M_{\sigma} U_{\sigma}=\operatorname{diag}\left\{\lambda_{\sigma}^{1}, \ldots, \lambda_{\sigma}^{N}\right\},
$$

where $\lambda_{\sigma}^{i} \geq 0, i=1, \ldots, N$. We denote $\bar{\xi}(t)=\left(U_{\sigma}^{T} \otimes I_{n}\right) \xi(t)$; then (18) can be rewritten as

$$
\begin{aligned}
\dot{V}(t) & =2 \bar{\xi}^{T}(t)\left[\left(I_{N} \otimes P A\right) \bar{\xi}(t)-\left(U_{\sigma}^{T} M_{\sigma} U_{\sigma}\right.\right. \\
& \left.\left.\otimes P B B^{T} P\right) \bar{\xi}(t-\tau)\right]+\bar{\xi}^{T}(t)\left(I_{N} \otimes P B B^{T} P\right) \bar{\xi}(t) \\
& -\bar{\xi}^{T}(t-\tau)\left(I_{N} \otimes P B B^{T} P\right) \bar{\xi}(t-\tau) \\
& =\sum_{i=1}^{N}\left[\bar{\xi}_{i}^{T}(t)\left(P A+A^{T} P+P B B^{T} P\right) \bar{\xi}_{i}(t)\right. \\
& -2 \lambda_{\sigma}^{i} \bar{\xi}_{i}^{T}(t) P B B^{T} P \bar{\xi}_{i}(t-\tau) \\
& \left.-\bar{\xi}_{i}^{T}(t-\tau) P B B^{T} P \bar{\xi}_{i}(t-\tau)\right]
\end{aligned}
$$

Note that, for any vectors $a, b \in R^{n}$ and any positive definite matrix $X \in R^{n \times n}$,

$$
-2 a^{T} b \leq \inf _{X>0}\left\{a^{T} X a+b^{T} X^{-1} b\right\} .
$$

Then we can obtain that

$$
\begin{aligned}
-2 \lambda_{\sigma}^{i} \bar{\xi}_{i}^{T}(t) P B B^{T} P \bar{\xi}_{i}(t-\tau) \\
\leq\left(\lambda_{\sigma}^{i}\right)^{2} \bar{\xi}_{i}^{T}(t) P B B^{T} P \bar{\xi}_{i}(t) \\
\quad+\bar{\xi}_{i}^{T}(t-\tau) P B B^{T} P \bar{\xi}_{i}(t-\tau) .
\end{aligned}
$$

Therefore, we have

$\dot{V}(t)$

$$
\begin{aligned}
& \leq \sum_{i=1}^{N} \bar{\xi}_{i}^{T}(t)\left[P A+A^{T} P+\left(1+\left(\lambda_{\sigma}^{i}\right)^{2}\right) P B B^{T} P\right] \bar{\xi}_{i}(t) \\
& \leq \sum_{i=1}^{N} \bar{\xi}_{i}^{T}(t)\left[P A+A^{T} P+\left(1+\bar{\delta}^{2}\right) P B B^{T} P\right] \bar{\xi}_{i}(t) \\
& \leq-\eta \sum_{i=1}^{N} \bar{\xi}_{i}^{T}(t) \bar{\xi}_{i}(t)=-\eta \sum_{i=1}^{N} \xi_{i}^{T}(t) \xi_{i}(t) .
\end{aligned}
$$

The assumption that the graphs are jointly connected across each time interval $\left[t_{i}, t_{i+1}\right)$ includes two cases. One case is that there is a subinterval $\left[t_{i}^{j}, t_{i}^{j+1}\right), 0 \leq j \leq m_{i}-1$, such that $\overline{\mathscr{G}}_{\sigma\left(t_{i}^{j}\right)}$ is connected; the other case is that all the graphs $\overline{\mathscr{G}}_{\sigma\left(t_{i}^{j}\right)}, 0 \leq j \leq m_{i}-1$, are disconnected. To derive our conclusion, we consider the two cases, respectively.

Case 1. There is a subinterval $\left[t_{i}^{j}, t_{i}^{j+1}\right), 0 \leq j \leq m_{i}-1$, such that $\overline{\mathscr{G}}_{\sigma\left(t_{i}^{j}\right)}$ is connected.
In this case, we know that $M_{\sigma\left(t_{i}^{j}\right)}$ is positive definite, which means $\eta_{j}>0$. By the definition of $\eta$, we get $\eta>0$. Let $\dot{V}(t) \equiv$ 0 ; we obtain that, during the subinterval $\left[t_{i}^{j}, t_{i}^{j+1}\right), \xi(t) \equiv 0$. Consider the initial-value problem for the equation

$$
\begin{aligned}
& \dot{\xi}(t)=\left(I_{N} \otimes A\right) \xi(t)-\left(M_{\sigma(t)} \otimes B K\right) \xi(t-\tau), \\
& \xi(t)=0, \quad t \in\left[t_{i}^{j}, t_{i}^{j+1}\right),
\end{aligned}
$$

where $t_{i}^{j+1}-t_{i}^{j} \geq \tau$. We know the unique solution is $\xi(t) \equiv 0$. By LaSalle's invariance principle, we conclude that $\lim _{t \rightarrow \infty} \xi(t)=0$, which implies that the closed-loop system is asymptotically stable.

Case 2. All the graphs $\overline{\mathscr{G}}_{\sigma\left(t_{i}^{j}\right)}, 0 \leq j \leq m_{i}-1$, are disconnected.

In this case, $\eta_{j}=0,0 \leq j \leq m_{i}-1$, which means $\eta=0$. Thus $\dot{V}(t) \leq 0$ and $\lim _{t \rightarrow \infty} V(t)$ exists. Based on the fact that $\dot{V}(t)$ is bounded below by zero, we denote $\lim _{t \rightarrow \infty} V(t)=$ $V_{0}$, where $V_{0} \geq 0$. In the following, we will prove that $V_{0}=0$. By contradiction, we suppose that $V_{0}>0$. Denote the maximum eigenvalue of positive definite matrix $P$ as $\alpha$, the maximum eigenvalue of matrix $P B B^{T} P$ as $\beta$, and the minimum eigenvalue of matrix $P B B^{T} P$ as $\gamma$. Without loss of generality, let $\|\xi(t-d)\|_{2} \leq \rho\|\xi(t)\|_{2}, d \in[\tau, 0]$, where $\rho$ is a given constant. We have

$$
\begin{aligned}
V_{0} & \leq V(t) \leq \alpha\|\xi(t)\|_{2}^{2}+\beta \rho^{2} \tau\|\xi(t)\|_{2}^{2} \\
& =\left(\alpha+\beta \rho^{2} \tau\right)\|\xi(t)\|_{2}^{2}
\end{aligned}
$$

that is, $\|\xi(t)\|_{2} \geq \sqrt{V_{0} /\left(\alpha+\beta \rho^{2} \tau\right)}, t \in[-\tau, \infty)$.

Note that

$$
\begin{aligned}
& \dot{V}(t) \leq \sum_{i=1}^{N} \bar{\xi}_{i}^{T}(t)\left[P A+A^{T} P+\left(1+\left(\lambda_{\sigma}^{i}\right)^{2}\right) P B B^{T} P\right] \\
& \cdot \bar{\xi}_{i}(t)=\sum_{i=1}^{N} \bar{\xi}_{i}^{T}(t) \\
& \cdot\left[P A+A^{T} P+\left(1+\bar{\delta}^{2}+\left(\lambda_{\sigma}^{i}\right)^{2}-\bar{\delta}^{2}\right) P B B^{T} P\right] \\
& \cdot \bar{\xi}_{i}(t)=\sum_{i=1}^{N} \bar{\xi}_{i}^{T}(t)\left[P A+A^{T} P+\left(1+\bar{\delta}^{2}\right) P B B^{T} P\right] \\
& \cdot \bar{\xi}_{i}(t)-\sum_{i=1}^{N} \bar{\xi}_{i}^{T}(t)\left(\bar{\delta}^{2}-\left(\lambda_{\sigma}^{i}\right)^{2}\right) P B B^{T} P \bar{\xi}_{i}(t) \\
& \leq-\eta \sum_{i=1}^{N} \bar{\xi}_{i}^{T}(t) \bar{\xi}_{i}(t)-\sum_{i=1}^{N} \bar{\xi}_{i}^{T}(t)\left(\bar{\delta}^{2}-\delta^{2}\right) \\
& \cdot P B B^{T} P \bar{\xi}_{i}(t) .
\end{aligned}
$$




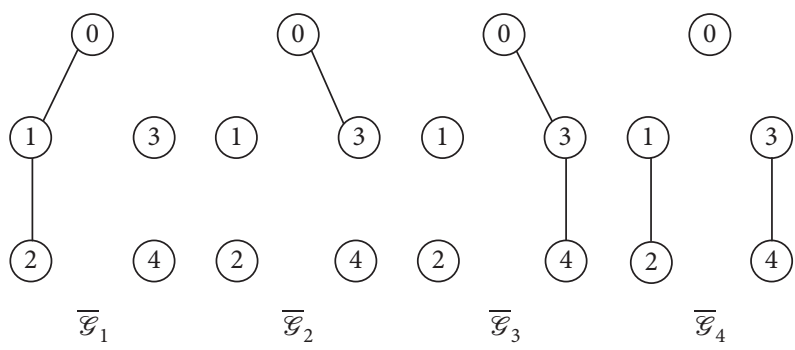

FIGURE 1: Interaction graphs.

With $\eta=0$ and $P B B^{T} P \geq \gamma I$, we have

$$
\begin{aligned}
\dot{V}(t) & \leq-\left(\bar{\delta}^{2}-\delta^{2}\right) \gamma \sum_{i=1}^{N} \bar{\xi}_{i}^{T}(t) \bar{\xi}_{i}(t) \\
& =-\left(\bar{\delta}^{2}-\delta^{2}\right) \gamma\|\xi(t)\|_{2}^{2} \\
& \leq-\left(\bar{\delta}^{2}-\delta^{2}\right) \gamma \frac{V_{0}}{\alpha+\beta \rho^{2} \tau} .
\end{aligned}
$$

Therefore, we can obtain that

$$
V(t) \leq V(0)-\left(\bar{\delta}^{2}-\delta^{2}\right) \gamma \frac{V_{0}}{\alpha+\beta \rho^{2} \tau} t
$$

which implies that $V(t)<0$ with $t$ sufficiently large. It is contrary to the fact that $V(t) \geq 0$. Thus, we conclude that $V_{0}=0$ and $\lim _{t \rightarrow \infty} \xi(t)=0$.

Remark 5. The feasibility problems of the Riccati inequalities have been well studied in the literature. Some of the notable results are summarized in $[15,16]$. The readers can refer to the above-mentioned excellent works on these subjects for a detailed exposition. For (6), a simple criterion is that if the pair $(A, B)$ is stabilizable and there are no eigenvalues of pure imaginary for $H=\left(\begin{array}{cc}A & \left(1+\delta^{2}\right) B B^{T} \\ -\eta I & -A^{T}\end{array}\right)$, then (6) is feasible. Also, we can get similar result for (16).

Remark 6. In recent years, consensus problems of high-order multiagent systems were investigated in the literature. Zhou and Lin [17] studied the consensus problem of high-order multiagent systems with time delays in both the communication network and inputs. He and Cao [18] generalized the second-order consensus algorithm to high-order systems and exact relationship between feedback gain and system parameters was established. Liu and Jia [19] considered output consensus problem of directed networks of multiple high-order agents with external disturbances. The results presented in this paper are totally different from those in [17-19], where fixed interaction topologies are studied. In our work, both switching interaction topologies and time delays are considered. Based on Riccati inequalities, algebraic conditions for consensus problem are established.

\section{Simulation Results}

In this section, we will give an example to illustrate the theoretical results. Consider a multiagent system consisting of a leader and four followers. The state of the leader is the dynamic target. The communication time delay is $\tau=0.1 \mathrm{~s}$. The system matrices are

$$
\begin{aligned}
& A=\left[\begin{array}{cc}
-0.2 & 3 \\
-2 & -0.2
\end{array}\right], \\
& B=\left[\begin{array}{lc}
6 & 0.3 \\
0 & 3
\end{array}\right] .
\end{aligned}
$$

Figure 1 shows the interaction graphs $\left\{\overline{\mathscr{G}}_{1}, \overline{\mathscr{G}}_{2}, \overline{\mathscr{G}}_{3}, \overline{\mathscr{G}}_{4}\right\}$. The communication topology of the multiagent system switches every $0.25 \mathrm{~s}$ as the sequence $\overline{\mathscr{G}}_{1} \rightarrow \overline{\mathscr{G}}_{2} \rightarrow \overline{\mathscr{G}}_{3} \rightarrow$ $\overline{\mathscr{G}}_{4} \rightarrow \overline{\mathscr{G}}_{1} \rightarrow \overline{\mathscr{G}}_{2} \rightarrow \overline{\mathscr{G}}_{3} \rightarrow \overline{\mathscr{G}}_{4} \rightarrow \cdots$. The initial values are set randomly. Obviously, the interaction graphs are jointly connected. In this section, we choose

$$
\begin{aligned}
P & =\left[\begin{array}{cc}
0.001 & 0 \\
0 & 0.0015
\end{array}\right], \\
K & =\left[\begin{array}{ll}
0.0059 & 0.0002 \\
0.0004 & 0.0045
\end{array}\right] .
\end{aligned}
$$

The state error trajectories of the agents are shown in Figure 2. From the figures, we can see that the agents follow the leader asymptotically.

\section{Conclusions}

In this paper, we consider the consensus problem of multiagent system with time delays by a leader-following approach. The state of the leader is used as a dynamic target of the followers. We use graph theory to describe the interaction topology. To solve this problem, we presented a local information protocol and proved that the consensus problem can be solved if there is a solution to a given Riccati inequality. A numerical example was introduced to indicate the correctness of our results.

\section{Competing Interests}

The authors declare that there is no conflict of interests regarding the publication of this paper. 


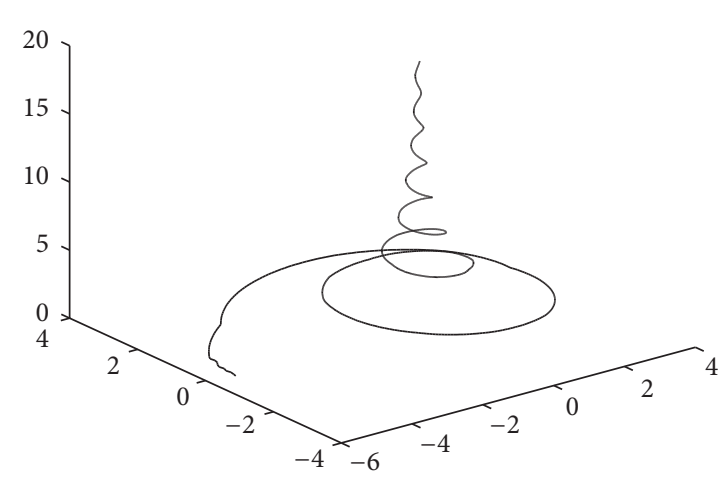

(a) Agent 1

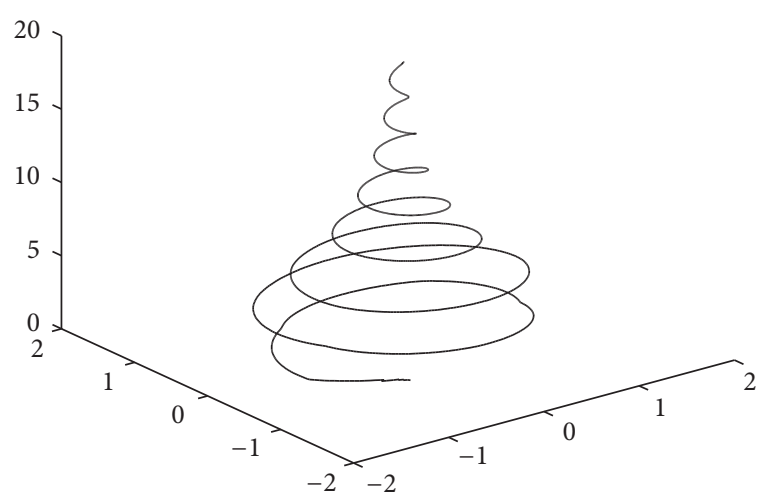

(b) Agent 2

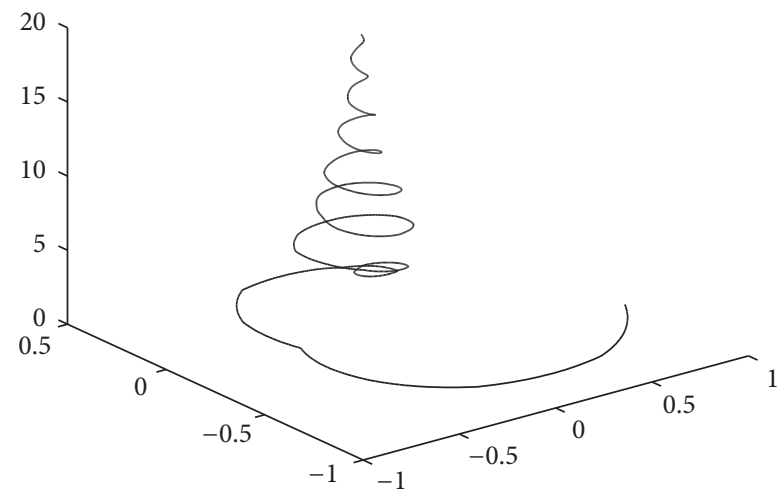

(c) Agent 3

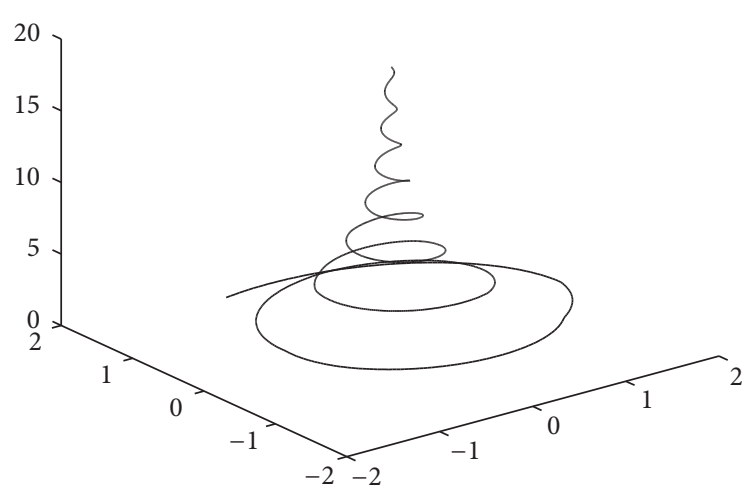

(d) Agent 4

FIgURE 2: The state error trajectories of the agents.

\section{Acknowledgments}

This work was supported by the National Natural Science Foundation of China (NSFC: 61603050) and the Fundamental Research Funds for the Central Universities.

\section{References}

[1] T. Vicsek, A. Czirok, E. Ben-Jacob, I. Cohen, and O. Shochet, "Novel type of phase transition in a system of self-driven particles," Physical Review Letters, vol. 75, no. 6, pp. 1226-1229, 1995.

[2] A. Jadbabaie, J. Lin, and A. S. Morse, "Coordination of groups of mobile autonomous agents using nearest neighbor rules," Institute of Electrical and Electronics Engineers. Transactions on Automatic Control, vol. 48, no. 6, pp. 988-1001, 2003.

[3] R. Olfati-Saber and R. M. Murray, "Consensus problems in networks of agents with switching topology and time-delays," IEEE Transactions on Automatic Control, vol. 49, no. 9, pp. 15201533, 2004.

[4] W. Ren and R. W. Beard, "Consensus seeking in multiagent systems under dynamically changing interaction topologies," Institute of Electrical and Electronics Engineers. Transactions on Automatic Control, vol. 50, no. 5, pp. 655-661, 2005.
[5] Y. G. Sun, L. Wang, and G. Xie, "Average consensus in networks of dynamic agents with switching topologies and multiple timevarying delays," Systems and Control Letters, vol. 57, no. 2, pp. 175-183, 2008.

[6] F. Xiao and L. Wang, "Consensus protocols for discrete-time multi-agent systems with time-varying delays," Automatica, vol. 44, no. 10, pp. 2577-2582, 2008.

[7] P. Lin, Y. Jia, and L. Li, "Distributed robust $\mathrm{H}_{\infty}$ consensus control in directed networks of agents with time-delay," Systems and Control Letters, vol. 57, no. 8, pp. 643-653, 2008.

[8] P. Lin and Y. Jia, "Consensus of a class of second-order multiagent systems with time-delay and jointly-connected topologies," IEEE Transactions on Automatic Control, vol. 55, no. 3, pp. 778-784, 2010.

[9] Z. Lin, B. Francis, and M. Maggiore, "Necessary and sufficient graphical conditions for formation control of unicycles," IEEE Transactions on Automatic Control, vol. 50, no. 1, pp. 121-127, 2005.

[10] I. D. Couzin, J. Krause, N. R. Franks, and S. A. Levin, "Effective leadership and decision-making in animal groups on the move," Nature, vol. 433, no. 7025, pp. 513-516, 2005.

[11] J. Hu and Y. Hong, "Leader-following coordination of multiagent systems with coupling time delays," Physica A, vol. 374, no. 2, pp. 853-863, 2007. 
[12] Y. Hong, J. Hu, and L. Gao, "Tracking control for multiagent consensus with an active leader and variable topology," Automatica. A Journal of IFAC, the International Federation of Automatic Control, vol. 42, no. 7, pp. 1177-1182, 2006.

[13] Y. Zheng and L. Wang, "Consensus of switched multiagent systems," IEEE Transactions on Circuits and Systems II: Express Briefs, vol. 63, no. 3, pp. 314-318, 2016.

[14] X. Lin and Y. Zheng, "Finite-Time Consensus of Switched Multiagent Systems," IEEE Transactions on Systems, Man, and Cybernetics: Systems, no. 99, pp. 1-11, 2016.

[15] Y. Jia, Robust H1 Control, Science Press, Beijing, China, 2007.

[16] Y. Liu, K. Yao, and Y. Lu, "Robust adaptive control for a longitudinal model of mars airplane," in Proceedings of the 10th IEEE International Conference on Control and Automation (ICCA '13), Hangzhou, China, June 2013.

[17] B. Zhou and Z. Lin, "Consensus of high-order multi-agent systems with input and communication delays-state feedback case," in Proceedings of the 1st American Control Conference (ACC '13), pp. 4027-4032, Washington, DC, USA, June 2013.

[18] W. He and J. Cao, "Consensus control for high-order multiagent systems," IET Control Theory \& Applications, vol. 5, no. 1, pp. 231-238, 2011.

[19] Y. Liu and Y. Jia, "Consensus problem of high-order multi-agent systems with external disturbances: an Ho analysis approach," International Journal of Robust and Nonlinear Control, vol. 20, no. 14, pp. 1579-1593, 2010. 


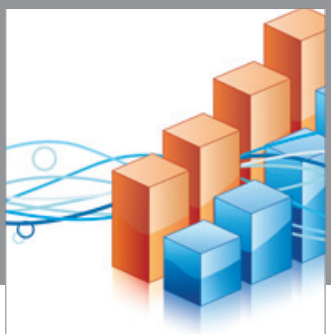

Advances in

Operations Research

vatem alat4

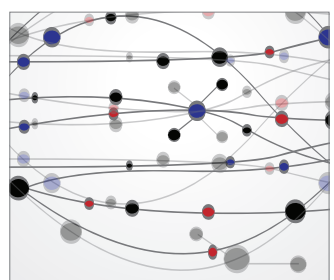

\section{The Scientific} World Journal
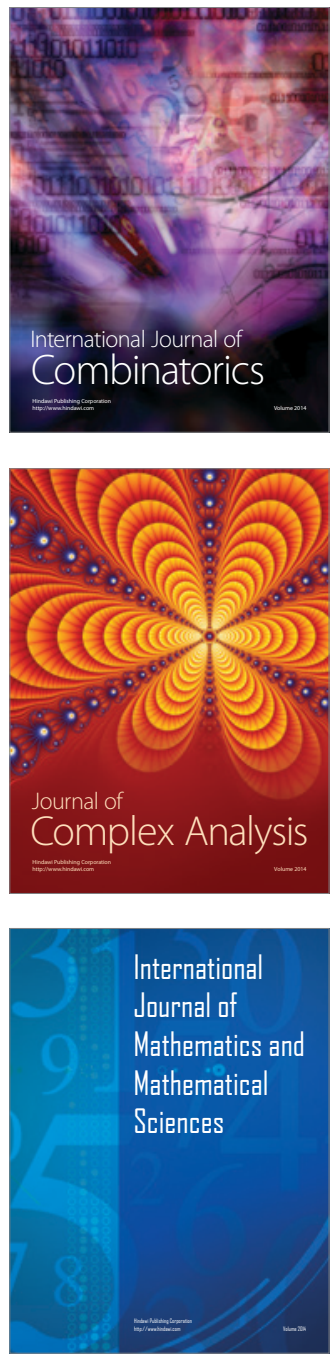
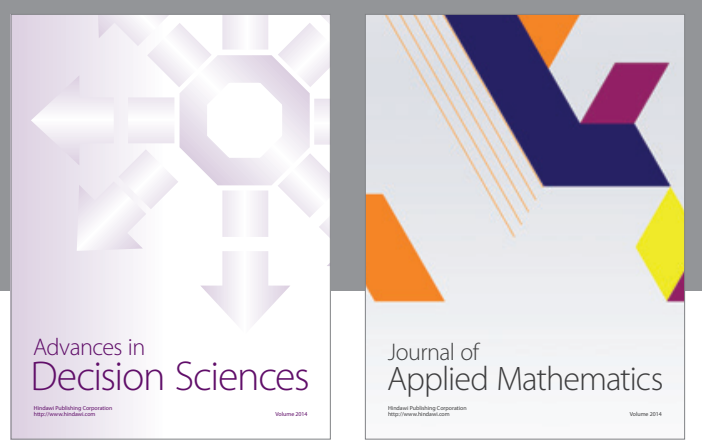

Algebra

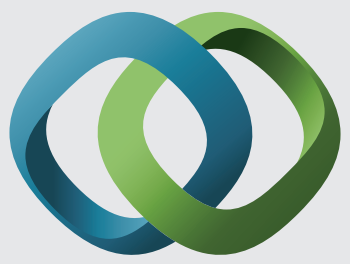

\section{Hindawi}

Submit your manuscripts at

https://www.hindawi.com
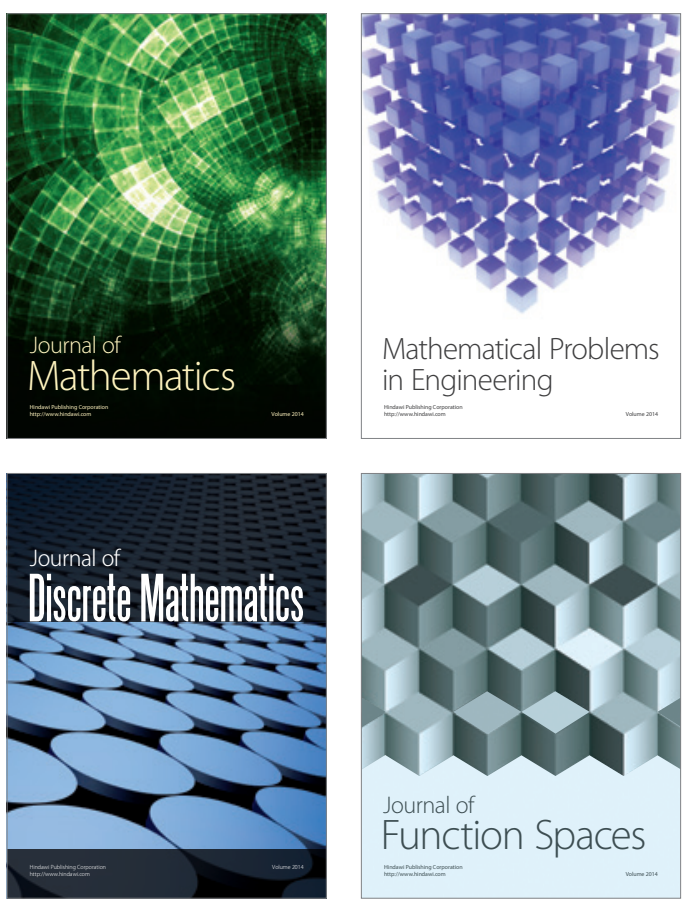

Mathematical Problems in Engineering
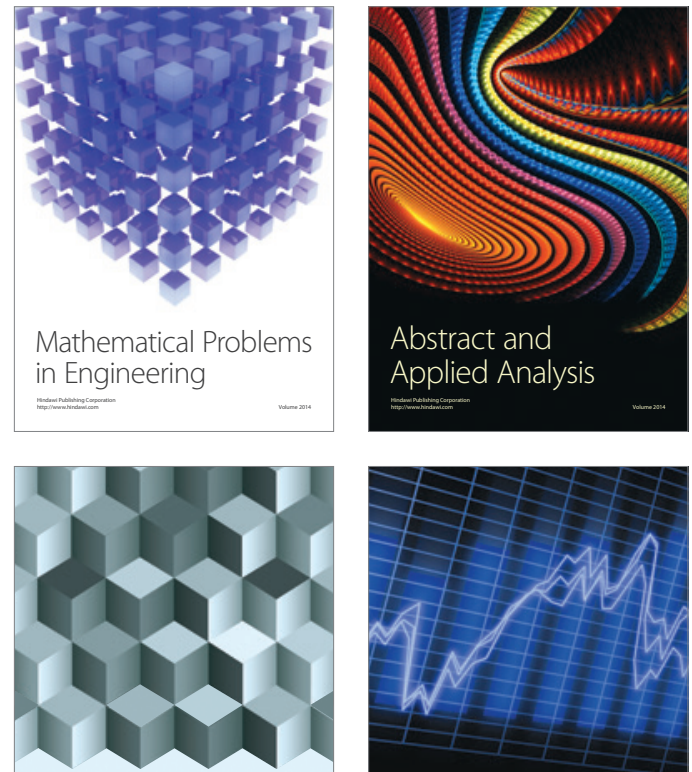

Journal of

Function Spaces

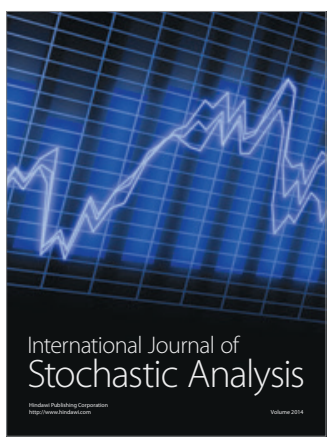

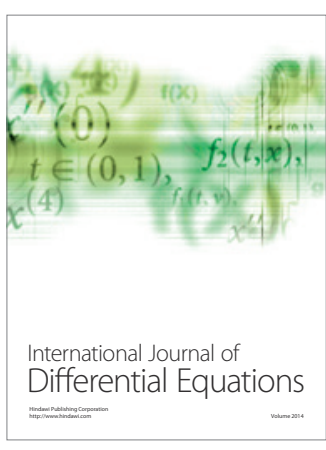
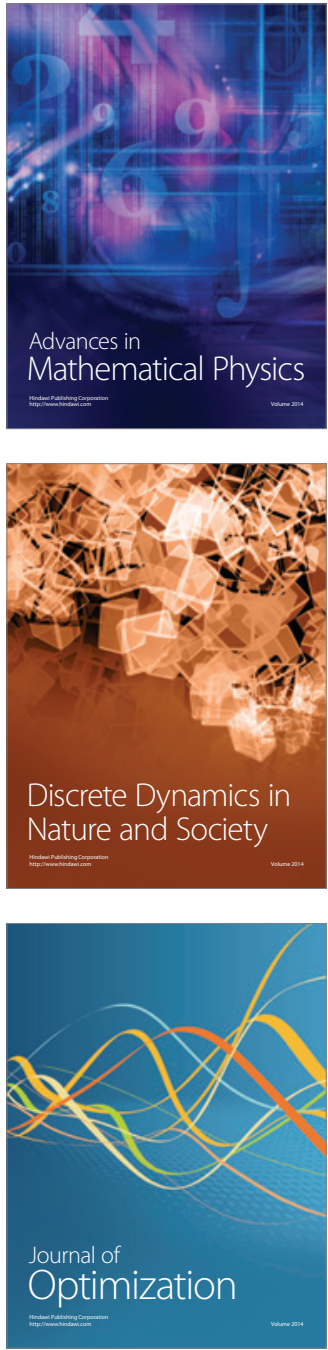